ARTICLE

\title{
A self-powered analog sensor-data-logging device based on Fowler-Nordheim dynamical systems
}

Darshit Mehta (1D ${ }^{1}$, Kenji Aono (1D ${ }^{2}$ \& Shantanu Chakrabartty (D) 1,2凶

Continuous, battery-free operation of sensor nodes requires ultra-low-power sensing and data-logging techniques. Here we report that by directly coupling a sensor/transducer signal into globally asymptotically stable monotonic dynamical systems based on Fowler-Nordheim quantum tunneling, one can achieve self-powered sensing at an energy budget that is currently unachievable using conventional energy harvesting methods. The proposed device uses a differential architecture to compensate for environmental variations and the device can retain sensed information for durations ranging from hours to days. With a theoretical operating energy budget less than 10 attojoules, we demonstrate that when integrated with a miniature piezoelectric transducer the proposed sensor-data-logger can measure cumulative "action" due to ambient mechanical acceleration without any additional external power.

\footnotetext{
${ }^{1}$ Department of Biomedical Engineering, Washington University in St. Louis, 1 Brookings Drive, St. Louis, MO 63130, USA. ${ }^{2}$ Department of Electrical and

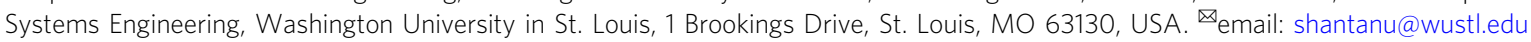


E or sensing systems like IoT devices or biomedical implants that operate in resource-constrained settings, utilizing a battery may be impractical due to biocompatibility concerns, size constraints or due to technical challenges involved in replacing the battery. Self-powered sensors (SPSs) can obviate the need for batteries by harvesting their operational energy directly from ambient sources, such as light ${ }^{1}$ or mechanical vibrations ${ }^{2}$. SPSs achieve this by first buffering ambient energy using standard power-conditioning techniques before activating the basic computation/sensing and sometimes telemetry functions ${ }^{3,4}$. However, when the objective is to sense and compute a simple function, like the total signal energy or a cumulative "action", an application specific but ultra-energy-efficient variant of SPS could be designed by combining the operational physics of signal transduction, rectification and non-volatile data storage. One such SPS was reported in refs. 5,6 where a cumulative measure of mechanical activity was sensed, computed and directly stored on floating-gate memories ${ }^{7}$. Similar techniques could also be applied to other non-volatile technologies for sensing the event of interest as an equivalent change in magnetoresistance in $\mathrm{MRAM}^{8}$, change polarization in $\mathrm{FeRAM}^{9}$, or change in electrical conductance in memristor-type systems ${ }^{10}$. However, these approaches require power conditioning such as rectification or voltage-boosting to meet the activation thresholds and to initiate the non-volatile state-change. Operational limits arise due to rectification efficiency, and due to material properties that influence diode thresholds or leakage currents. Note that some energy harvesting systems report low voltage continuous operation (i.e., $<50 \mathrm{mV}$ ); however, they require higher activation thresholds for initial start-up conditions (e.g., $>600 \mathrm{mV})^{11-13}$.

We propose a self-powered sensing system, where instead of harvesting the energy to switch between static memory states, the sensing signal is used for modulating a synchronized dynamic state. In this regard, dynamical systems, both natural and artificial, have been shown to store information in their dynamic states $^{14-16}$. In this work, we show the feasibility of this approach for self-powered sensing and data logging, but at chip-scale. This is illustrated in Fig. 1a, which shows two synchronized globally asymptotically stable (G.A.S.) dynamical systems; a sensing system and a reference system. A time-varying input signal modulates the state trajectories of the sensing dynamical system leading to its desynchronization with respect to the reference dynamical system. The relative degree of desynchronization between the two systems serves as a medium for sensing and storing the cumulative effect of the input modulation. While the principle is relatively straightforward, there exist two key challenges in implementing the proposed concept at a chip-scale. First, due to self-powering requirements, the synchronized G.A.S. dynamical system can only be implemented using leakage processes driven by intrinsic thermal or quantum transport of electrons. The simplest of such a system can be modeled by an equivalent circuit shown in Fig. 1b. The capacitor $C$ in the circuit models the dynamical state (denoted by the time-varying voltage $V_{t}$ ) and the time-dependent system trajectory is determined by a leakage current $I\left(V_{t}\right)$. The capacitor $C_{\text {in }}$ couples the input signal $x_{t}$ into the dynamical system. The challenge is that an ultra-low leakage current $I\left(V_{t}\right)$ is required to ensure that the dynamical system is operational for the duration of sensing and data logging. For instance, a $1-\mathrm{V}$ change across a $1-\mathrm{pF}$ on-chip capacitor over a duration of 1 day would require a leakage current of 10 attoamperes. Even if it were possible to implement such low-leakage currents, it is difficult to ensure that the magnitude of the currents match across different devices to ensure state synchronization. The second challenge with regard to data logging is that there exists a trade-off between the non-linearity in the dynamical systems response and the duration over which the information can be retained. As shown in Supplementary Fig. 1 if a constant leakage element (for example, reverse leakage current) is used, not only do the system trajectories rapidly converge to the final steady state, but the modulation signal does not cause a change in the sensing system trajectory with respect to the reference system trajectory. On the other hand, a resistive or a direct-tunneling leakage element will be sensitive to the changes in modulation signal but will be unable to keep the two trajectories separated for long periods of time, leading to low retention time. In this report, we show that a differential G.A.S. dynamical system ${ }^{17}$

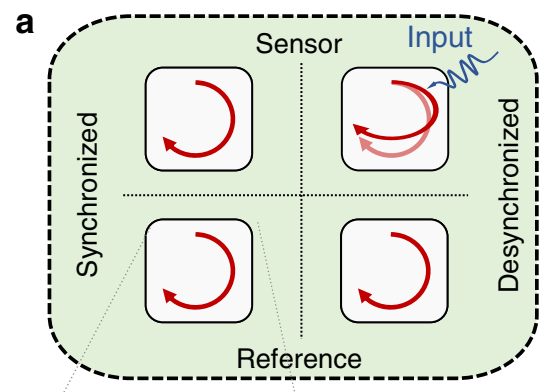

d

b

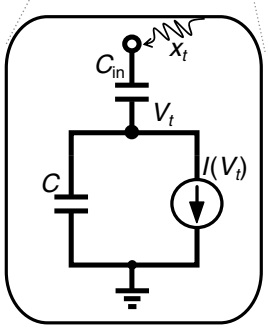

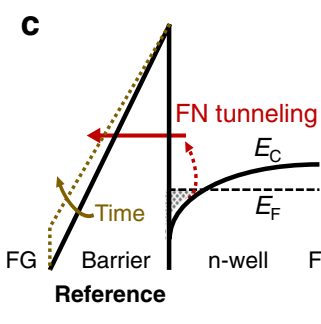
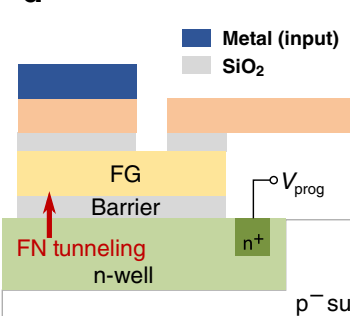

Poly

Poly2 Doped Si
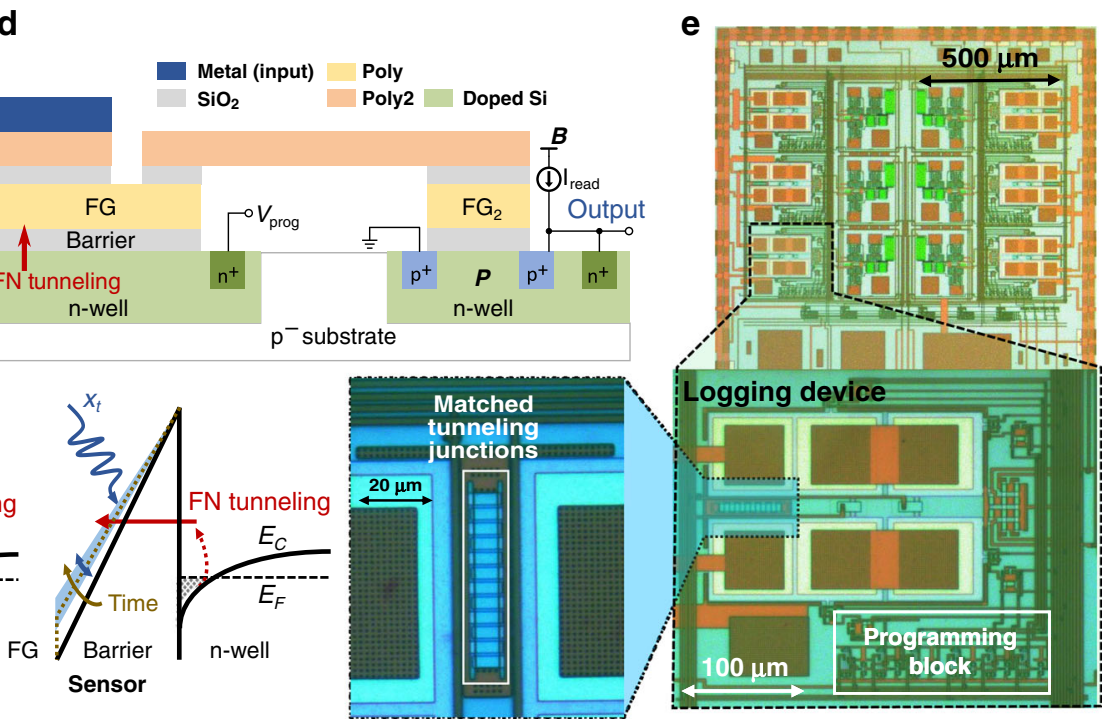

Fig. 1 Operating principle and architecture of the proposed sensor-data logger. a Principle of sensing and data logging where the input signal leaves its trace on a pair of synchronized dynamical system through a desynchronization process. b Equivalent circuit model of a self-powered dynamical system where the charge on a capacitor $C$ stores the dynamical state of the system and the dynamics is governed by a leakage current $I\left(V_{t}\right)$ and ambient stimuli $x_{t}$. c Band diagram corresponding to the tunneling junction where the electrons tunnel across the triangular energy barrier and the input signal $x_{t}$ modulates the barrier shape. $\mathbf{d}$ Cross-section of the sensor-data-logging device showing the FN tunneling junction, the floating gate which is coupled to a read-out transistor $P$ and a buffer $B$. e Micrograph of the fabricated devices, with inset showing a pair of dynamical systems configured in a differential architecture. 
implemented using a Fowler-Nordheim (FN) quantum tunneling device $^{18}$ can address all these challenges.

\section{Results}

Differential FN tunneling device acts as a long-term reliable synchronized dynamical system. The operating physics of an FN quantum-tunneling-based dynamical system is illustrated using an energy-band diagram in Fig. $1 \mathrm{c}^{19}$. From a practical point-ofview, this energy-band configuration can be achieved across a thermally grown gate-oxide (silicon-di-oxide), which acts as an FN tunneling barrier that separates a lightly doped n-type semiconductor substrate from an electrically insulated but conductive polysilicon island (labeled as a floating gate). A twodimensional electron gas and a triangular FN tunneling barrier (as shown in Fig. 1c) is created by initiating a large potential difference across the semiconductor-floating-gate interface. Thermally excited electrons then tunnel through the triangular FN tunneling barrier onto the floating gate (FG) and cannot escape due to the surrounding electrical insulation. Each electron that tunnels through the barrier, and is retained, changes the potential of the floating gate, which in turn decreases the slope of the FN tunneling barrier (shown in Fig. 1c). Figure 1d shows the cross-section of such an FN tunneling device, whereby the floating gate is coupled to a programming transistor $P$ and a source follower buffer $B$. The read-out procedure and the procedure to initialize the charge on the floating gate is described in the "Methods" section and in Supplementary Fig. 2. In ref. ${ }^{18}$, we showed that the continuous-time dynamics of this device can be modeled using a first-order differential equation which results in the change in floating-gate voltage $V_{t}$ at time-instant $t$ as

$$
V_{t}=\frac{k_{2}}{\log \left(\mathrm{k}_{1} \mathrm{t}+\mathrm{k}_{0}\right)}+k_{3},
$$

where $k_{0}-k_{3}$ are model parameters. The parameters $k_{1}$ and $k_{2}$ depend on the area of tunneling junction, capacitance, temperature and material properties and the device structure, the parameter $k_{3}$ depends on the read-out mechanisms and the parameter $k_{0}$ depends on the initial conditions. For the proposed sensor-data logger, we employ a differential configuration as shown in Fig. 2a. The initial voltage (equivalently, charge) on each floating gate is precisely programmed through a combination of tunneling and hot-electron injection (see "Calibration" and "Initialization" in "Methods") ${ }^{20}$. One of the FN device's (labeled as the sensor) dynamics is modulated by an input signal $x_{t}$, and its desynchronization is measured with respect to a reference FN device as:

$$
\hat{Y}_{t}=V_{t}^{R}-V_{t}^{S} \text {. }
$$

Here, $V_{t}^{S}$ and $V_{t}^{R}$ refer to the sensor and reference floating-gate voltages, respectively. A capacitive divider (formed by $C_{\mathrm{c}}$ and $C_{\mathrm{FG} 2}$ ) followed by a source-follower is used to read out the floating-gate potential through the output node as shown in Fig. 2a. The floating node formed at the capacitive divider is independently programmed to a lower value $(\approx 3 \mathrm{~V})$ to ensure low probability of unwanted tunneling or injection through the transistor $\mathrm{FG}_{2}$. The outputs of the sensor and reference nodes, $V_{t}^{\text {sensor }}$ and $V_{t}^{\text {ref }}$, respectively, are measured using an external data acquisition system (Keithley DAQ6510) and shown in Fig. 2b. The differential output $Y_{t}$ in Fig. 2a is measured with respect to the initial value as

$$
Y_{t}=\left(V_{t}^{\text {ref }}-V_{t}^{\text {sensor }}\right)-\left(V_{0}^{\text {ref }}-V_{0}^{\text {sensor }}\right)=\Delta V_{t}^{\text {ref }}-\Delta V_{t}^{\text {sensor }} \propto \hat{Y}_{t} .
$$

For calculating $Y_{t}$, we use the change from their initial voltages at time-instant $t=0 \mathrm{~s}$ ( $\Delta V_{t}$ in Fig. 2c) to eliminate the offset in the read-out stage.
For each device, $<1 \%$ deviation was observed across trials, demonstrating the reliability of the tunneling dynamics and the reliability of the measurement setup. With respect to the differential measurements, $Y_{t}$ should be $0 \mathrm{~V}$ in a perfectly synchronized system. However, due to device mismatch and due to differences in the initialization procedure, we observe a baseline drift across all trials. This manifests as variations in device parameters $k_{1}-k_{3}$, which were estimated by regressing Eq. (1) to the empirical data (Supplementary Table 1). The estimated parameters were then used to compensate for drift and to determine the sensor output (Supplementary Fig. 3). Post-drift corrections are shown in Fig. 2d, which shows the maximum difference between a pair of trials to be $<300 \mu \mathrm{V}$. We measured the desynchronization of the differential FN device across temperatures ranging from $5^{\circ}$ to $40^{\circ}$. Higher temperatures led to faster tunneling, which led to a larger variation in $\Delta V_{t}$ within the range of $200-260 \mathrm{mV}$ as a function of temperature (Fig. 2e). Despite this variation, the measured desynchronization $Y_{t}$ had a significantly lower variance with standard deviation below $1 \mathrm{mV}$. These results show that the differential architecture is capable of compensating for variations in temperature. Note that an incorrect initialization of the reference device with respect to the sensor device will make the temperature compensation less robust, as shown by an outlier in Supplementary Fig. 4.

A simple behavioral model explains the data-logging principle. In the "Methods" section, we have derived a tractable mathematical model for the data sensed and stored by the sensor-data logger in response to an arbitrary time-varying input signal $x_{t}$. We found that the output of the data logger $Y_{T}$ measured at timeinstant $T$ can be expressed as

$$
Y_{T}=R(T) A_{x}(T),
$$

where $A_{x}(T)$ represents the total "action" due to the input signal $x_{t}$ accumulated up to the time instant $T$ and $R(T)$ is a "forgetting" factor that is independent of the input signal $x_{t} . R(T)$ models the data retention capability and arises due to resynchronization of the sensor and reference FN devices, after the sensor device is perturbed by $x_{t}$. In the "Methods" section, we show that the action $A_{x}(T)$ can be expressed in terms of device parameters as

$$
A_{x}(T)=\frac{k_{1}}{k_{2}} V_{0}^{2} \exp \left(\frac{-k_{2}}{V_{0}}\right) \int_{0}^{T}\left[\left(1+\frac{C_{\mathrm{R}} x_{t}}{V_{t}}\right)^{2} \exp \left(\frac{k_{2} C_{\mathrm{R}} x_{t}}{V_{t}\left(V_{t}+C_{\mathrm{R}} x_{t}\right)}\right)-1\right] \mathrm{d} t,
$$

and the resynchronization term $R(T)$ can be expressed as

$$
R(T)=\frac{V_{t}^{2}}{V_{0}^{2}} \exp \left(\frac{k_{2}}{V_{0}}-\frac{k_{2}}{V_{T}}\right) .
$$

Here $V_{t}$ is given by Eq. (1) with $V_{0}$ and $V_{T}$ representing the device voltage at time-instant $t=0$ and $t=T$ seconds. The parameter $C_{\mathrm{R}}$ in Eq. (5) models a capacitive divider that is formed due to the coupling of the input capacitance onto the floating gate. The Supplementary Figs. S5 and S6 show several examples of signals $x_{t}$ for which the first-order action model given by Eq. (4) accurately tracks a more computationally intensive ordinary differential equation (ODE)-based device model. In Supplementary Fig. 7, we show the "action" $A_{x}(T)$ corresponding to different signal types with different magnitude and energy. The results show that $A_{x}(T)$ is monotonic with respect to energy and hence can be used as a measure of cumulative energy.

In our controlled experiments, we subjected the FN datalogging device to a square pulse of varying magnitude but with a fixed duration of $120 \mathrm{~s}$. This duration was chosen because it is sufficiently long enough to elicit a measurable response and for 

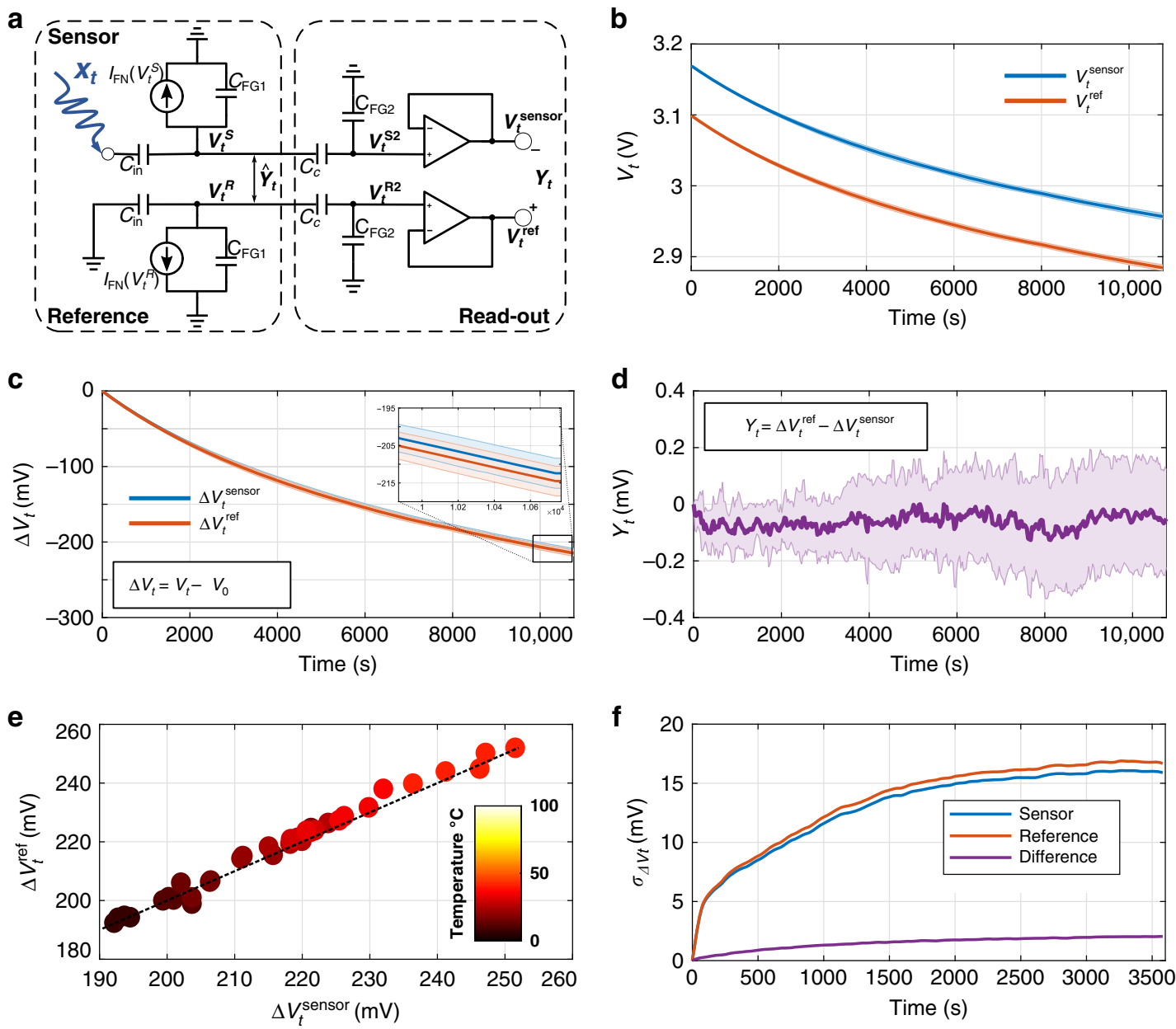

Fig. 2 Differential FN sensor-data-logging device and its measured response. a Equivalent circuit of the differential FN device coupled to the read-out circuitry. b Sensor and reference output voltages measured across nine trials after the device is initialized. c Change in sensor and reference values compared to the initial value $V_{0}$ as $\Delta V_{t}=V_{t}-V_{0}$. Shaded region in inset shows \pm 1 standard deviation. $\mathbf{d}$ Measured desynchronization between the sensing and reference devices, with bold line showing mean across trials. e Synchronization measured across a range of operating temperatures (5-40 ${ }^{\circ}$. The gradient (dark red to yellow) denotes an increase in operating temperature. $\mathbf{f}$ Standard deviation measured for the sensor, reference and the difference over 36 trials and across range of operating temperatures.

the purpose of device characterization. Also, the pulse was applied at a fixed time $(1800 \mathrm{~s})$, after which the desynchronization $Y_{T}$ was measured at different values of measurement time T. Experiments were conducted over a duration of $10,800 \mathrm{~s}(3 \mathrm{~h})$, with the datalogger responses measured every $30 \mathrm{~s}$. Each data logger was calibrated to similar initial conditions for all experiments wherein the sensor and the reference nodes were initialized to equal tunneling rates. A typical experiment demonstrating the recorder in operation is shown in Fig. 3a, which matches the model described in the "Methods" section. The RMSE between the model and measured data is $61 \mu \mathrm{V}$ with an $R^{2}$ of 0.9999 .

Measurement results across three repeated trials for input signals of magnitude $100 \mathrm{mV}$ and $-100 \mathrm{mV}$ are shown in Fig. $3 \mathrm{~b}$. The $100 \mathrm{mV}$ signal resulted in a sensor response of $0.8-1.5 \mathrm{mV}$ for the three data-logging devices. At the end of $3 \mathrm{~h}$, due to resynchronization, the sensor response decreases down to $0.5-0.6$ $\mathrm{mV}$. For the $-100 \mathrm{mV}$ input, responses after the modulation were in the range of -0.5 to $-0.9 \mathrm{mV}$, which dropped to -0.2 $\mathrm{mV}$ at the end of $3 \mathrm{~h}$. Though the three recorders had different responses, they were consistent across trials for the same recorder. The device responses at the end of $3 \mathrm{~h}$ for input signals of different magnitudes are shown in Fig. 3c. From the figure, it is evident that the data-logging device response is similar to a rectifier as summarized by the action model in Eq. (5). The action model fits the data for this wide range of input conditions with an $R^{2}$ of 0.9855 .

Self-powered operation of the proposed device. The selfpowered dynamical system created by FN tunneling leakage implies that the device can operate without any external power source, once initialized. We have verified this mode of operation by first disconnecting the sensor-data logger from any power supplies and then applying an external signal as an input. The experimental protocol and representative results are shown in Fig. 4a. Immediately after powering on the system, the output of the reference node was measured to be lower than the value predicted by the model given by Eq. (1). However, the measurement stabilized within $200 \mathrm{~s}$ and the output closely matched the model for the rest of the experiment, indicating that FN tunneling dynamics were conserved in the self-powering mode. Additionally, desynchronization between sensor and reference nodes was observed immediately after power was turned on, indicating that the external signal "acted" on the sensing node. Errors introduced during the stabilization period were consistent between the sensor and reference nodes-the differential architecture attenuated these errors. The magnitude of the response is an exponential function of the input signal magnitude (Fig. 4b) as 
a
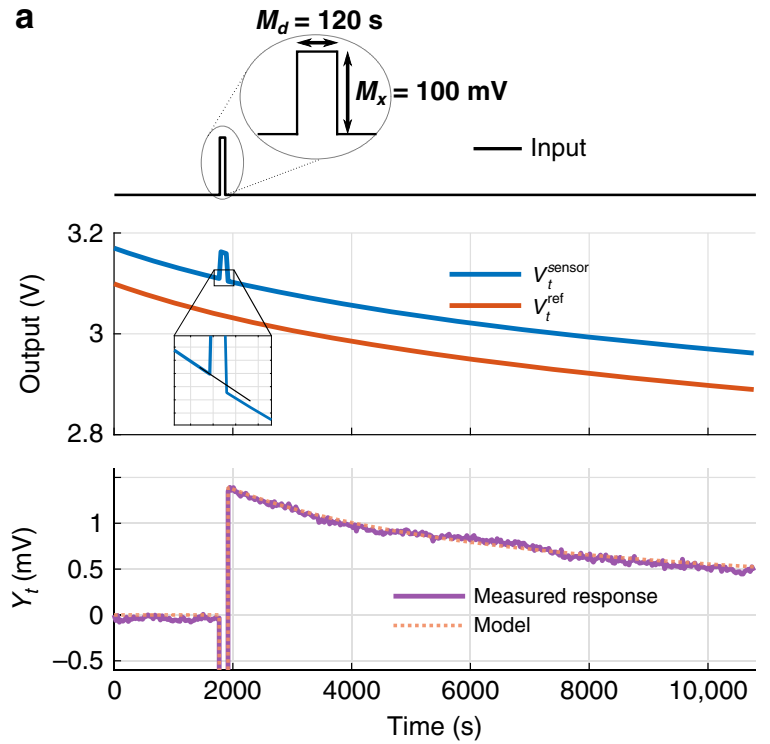

b

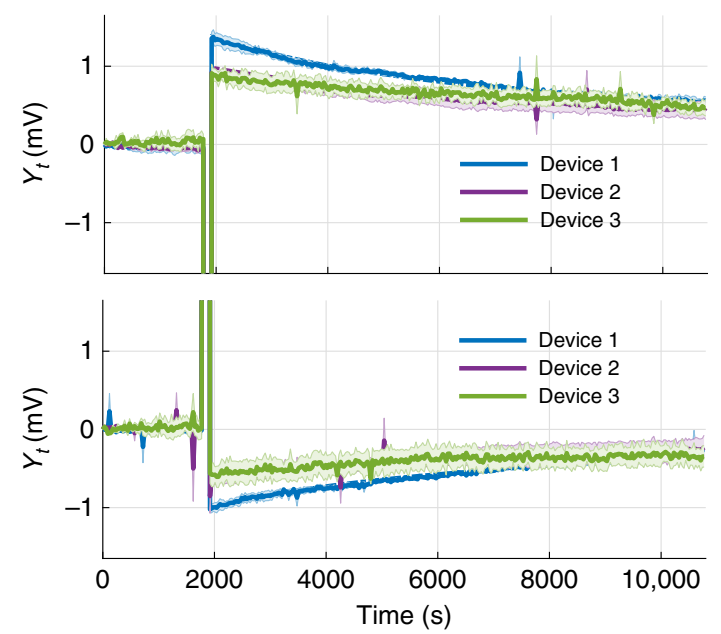

C

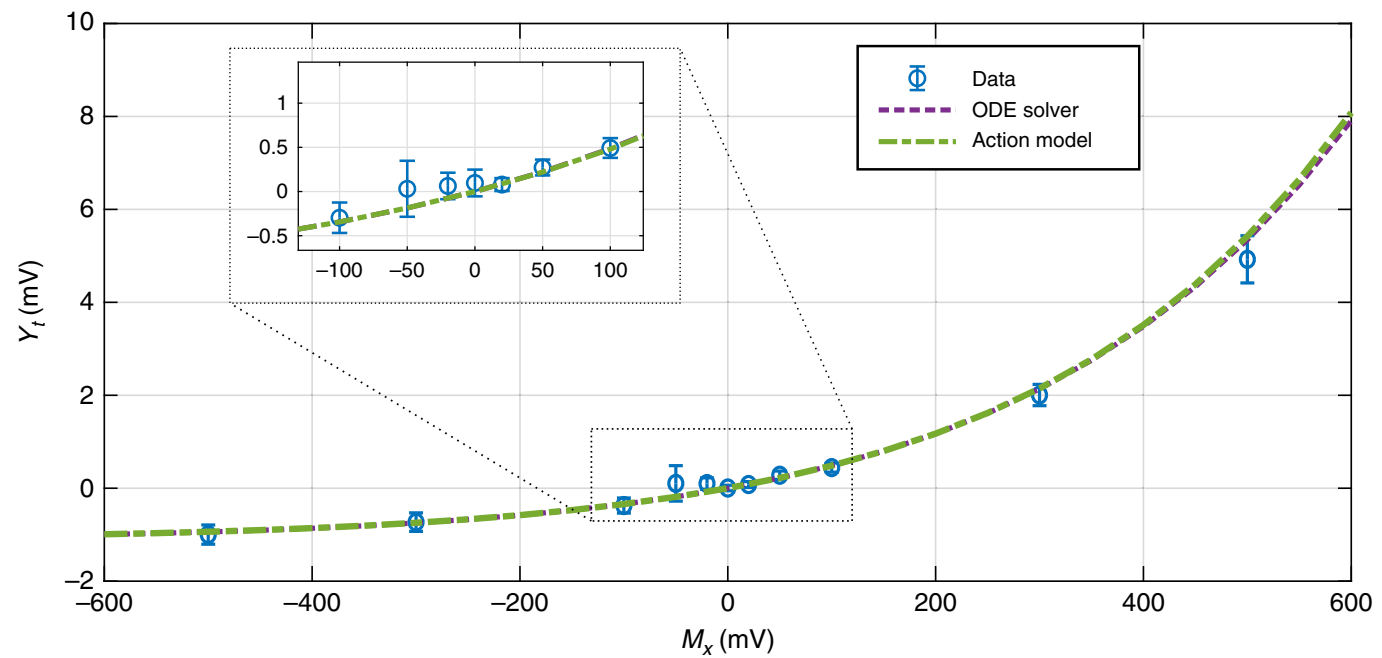

Fig. 3 Rectifying response of the sensor-data-logger device. a Output measured from the device when subjected to an input pulse. During the positive half of the input pulse, the tunneling rate increases and desynchronizes the sensor device with respect to the reference device. $\mathbf{b}$ Responses measured from three loggers across three trials. The loggers were initialized to different conditions, hence the difference in their measured responses. c Sensor responses for input signals over a range of amplitudes. Responses follow an exponential model, which can be accurately modeled by the action model and an ODE solver.

predicted by the action model of Eq. (5). Similarly, the recorder was able to record the number (and thus the energy) of discrete pulses applied (Fig. 4c) in the power-off state. The mean absolute error between model and observed data was $0.7 \mathrm{mV}$, higher than the errors obtained for continuously powered case (Fig. $4 \mathrm{~d}$ ).

Energy budget, sensing and retention limits. The rectification property of the FN data-logging device can be useful for measuring and logging the intensity of a time-varying signal like biopotentials or accelerometer output. The device is sensitive to input signals of any intensity since there is no threshold requirement on the input signal to activate the sensor. The caveat being, the data retention times for small magnitude signals will be shorter due to the resynchronization (modeled by $R(T)$ in Eq. (6)) and operational noise in the recorder. In a perfectly matched differential system, and in the absence of any input, the device response should be exactly $0 \mathrm{~V}$, because of synchronization. However, environmental factors, mismatch between the sensing and reference nodes, or stochasticity in tunneling mechanism, cause desynchronization and the recorder response deviates from the baseline. In general, the variance in the output increases with time (see Fig. $2 \mathrm{~d}$ for example). This increase in variance over time is a form of operational noise in the system $\left(\sigma_{t}\right)$. A model for $\sigma_{t}$ could be estimated by letting the recorder operate with respective inputs connected to the ground (similar to input referred noise experiments) and measuring the deviation of output from the baseline. Another source of noise is the readout noise $\left(N_{0}\right)$ which limits the resolution to which charge on the floating gate can be measured. Total noise $\left(N_{t}\right)$ is the sum of these two noise sources. While noise increases with time, recorder response decreases due to resynchronization. For a signal of given action, there will be a time instance $T_{\text {ret }}$, beyond which the signal-to-noise ratio (SNR) goes below a chosen threshold and input signal cannot be reconstructed with a desirable degree of certainty. We chose unity as our threshold for SNR, and we defined data retention as the time at which the signal falls below system noise. The Supplementary Fig. 8a shows via an illustration how data retention capacity for a given noise model can be estimated. The Supplementary Fig. $8 \mathrm{~b}$ shows that data retention capacity increases exponentially with the signal action. For a $10-\mathrm{mV}$ action, we 
a
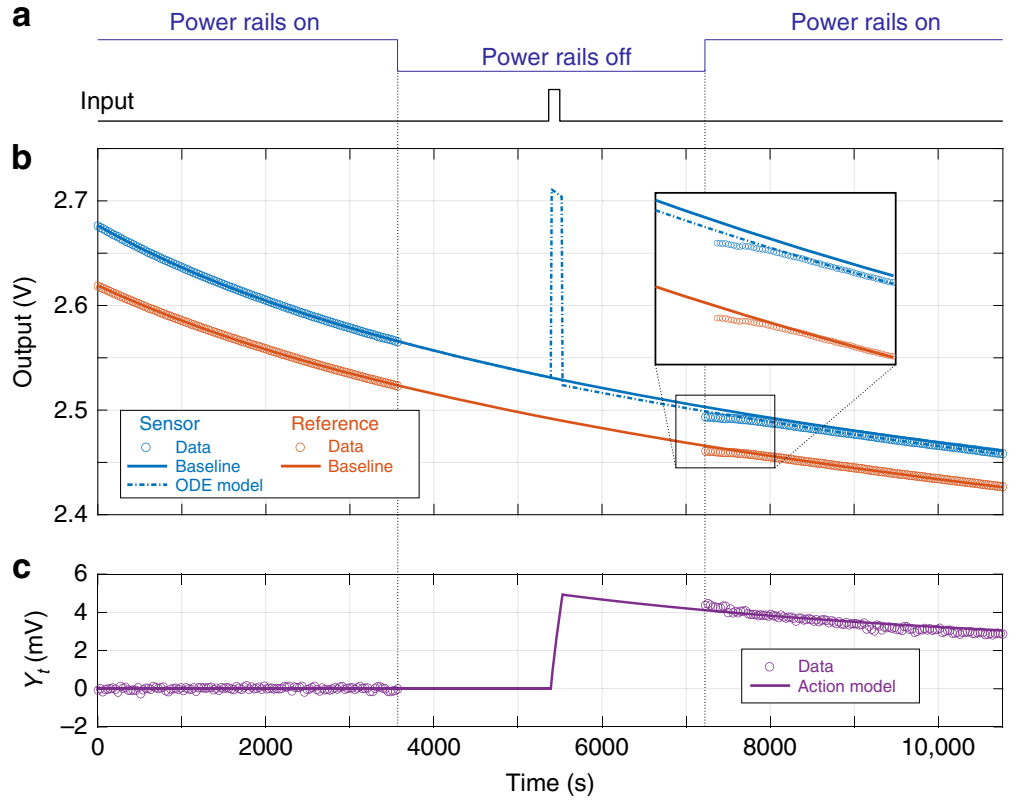

d
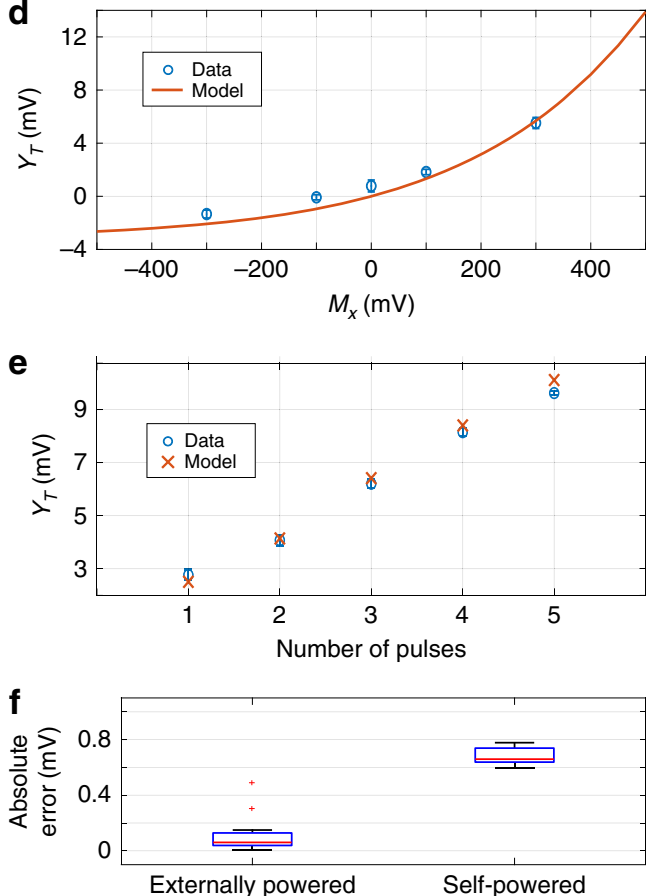

Fig. 4 Verification of the proposed device for self-powered sensing and data logging. a Power to the system is switched off at the 1-h mark, and then turned back on at 2-h mark. The input pulse is applied at the 1.5-h mark for a duration of $120 \mathrm{~s}$. b, c Output measured from the recorder when the power is $\mathrm{ON}$ and the comparison with the predicted model showing the process of desynchronization. $\mathbf{d}$ Recorder responses for input signals over a range of amplitudes. Responses follow an exponential model, which can be modeled by the action model. e Recorder responses for varying number of pulses (400 $\mathrm{mV}$ magnitude, $50 \mathrm{~s}$ duration each). $\mathbf{f}$ Distribution of absolute errors between measured data and model predictions, for externally powered and selfpowered cases estimated across all experiments.

could expect to measure significant deviation from baseline for over $300,000 \mathrm{~s}$ ( $\approx 4$ days). The action model can be used to estimate the energy-budget requirement on the sensing signal. Since the average FN tunneling current is $10^{-17} \mathrm{~A}$, the energy budget is less than an attojoule. Note that this is the energy to trigger desynchronization. However, isolating the energy dissipated due to FN tunneling from other energy dissipation factors is challenging because the FN tunneling current is on the order of attoamperes, which is orders of magnitude smaller than the reactive current generated by the transducer and the leakage current flowing through ESD protection diodes. In the Supplementary Note 9, we estimate the energy budget when the proposed sensor-data logger is driven by an arbitrary sensor signal.

Noise in the system can also be described by the effective number of bits (ENOB) (Supplementary Fig. 8c). For an assumed action range of $10 \mathrm{mV}, 10$ bits precision can be initially expected in a system with $10 \mu \mathrm{V}$ readout noise. In a perfectly matched system, ENOB would drop to 0 at $\approx 2 \times 10^{6} \mathrm{~s}$ (total recorder lifetime), but with the added operational noise it takes $\approx 3 \times 10^{5} \mathrm{~s}$ to reach 0 . Readings from multiple recorders can be combined to increase the ENOB of the system.

Self-powered sensing of action due to ambient acceleration. In this section, we demonstrate the use of the proposed sensor-data logger for battery-free sensing of ambient acceleration. We chose a piezoelectric transducer for sensing mechanical acceleration and for directly powering the sensor-data-logger device. Note that in this regard, other transducers, for e.g., photodiodes, RF antennas, thermocouples, could also be directly interfaced to the FN datalogging device to create other self-powered sensing modalities. A schematic of the experimental setup is shown in Fig. 5a. A polyvinylidene difluoride (PVDF) cantilever (TE Connectivity's Measurement Specialties MiniSense 100 Vibration Sensor with nominal resonant frequency - $75 \mathrm{~Hz}$ ) was mounted on a benchtop vibration table (3B Scientific Vibration Generator - U56001) that is externally actuated by a function generator. The table was actuated at an off-resonant frequency of $72 \mathrm{~Hz}$ for a range of actuating amplitudes. We simultaneously measured acceleration using a 3-axis accelerometer (Adafruit LIS3DH accelerometer) to use as the ground truth. Results are shown in Fig. 5b, c. We observed significant responses for vibration signals down to an acceleration of $0.0052 \mathrm{~g}\left(0.05 \mathrm{~m} / \mathrm{s}^{2}\right)$. For context, a refrigerator vibrates with an acceleration of around $0.1 \mathrm{~m} / \mathrm{s}^{2}$ (ref. ${ }^{21}$ ). The expected maximum output power of the piezoelectric sensor is on the order of tens of nanowatts of which only a fraction is used by the recorder to store the information. In the final experiment, we electrically disconnected all power to the recording system at the 1 -h (3600 s) mark, actuated the vibration table at $1.5 \mathrm{~h}(5400 \mathrm{~s})$ and reconnected the system at $2 \mathrm{~h}(7200 \mathrm{~s})$ to read out the output of the data logger. We observed vibration-induced desynchronization in this set of experiments as well, with the deviation as expected based on the earlier characterization tests.

\section{Discussion}

In this report, we proposed a novel method for designing an ultra-energy-efficient sensor-data-logging device, where the energy of the sensing signal is used to modulate the state trajectories of a synchronized dynamical system. We showed that an FN quantum tunneling device ${ }^{18}$ can be used to implement the proposed sensor-data-logger on a standard silicon process.

Our modeling study summarized in Supplementary Fig. 9 shows that there are multiple parameters, both operational and design parameters, that affect the retention time (or resynchronization) of the FN device. Change in any parameter, that increases (decreases) the "action" of the signal, would also lead to faster (slower) resynchronization. Thus, its net effect on the 

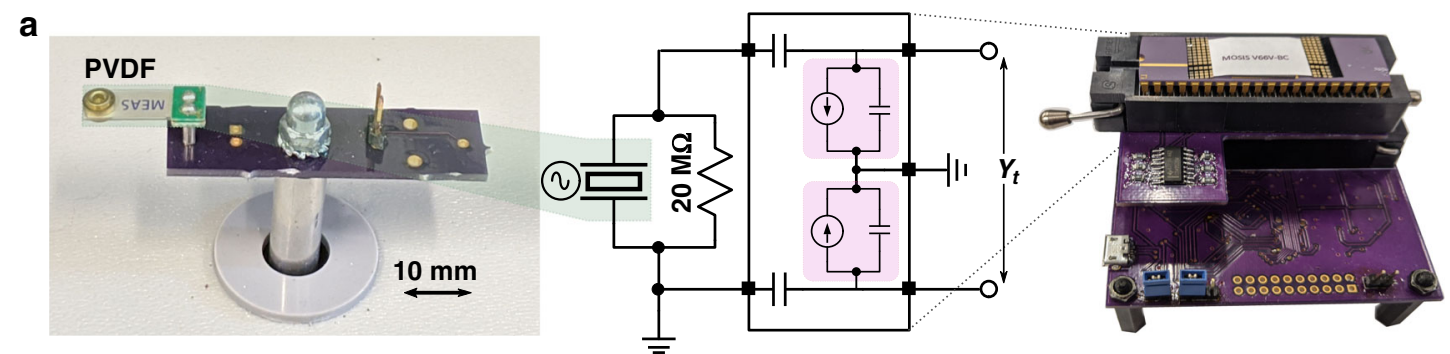

b

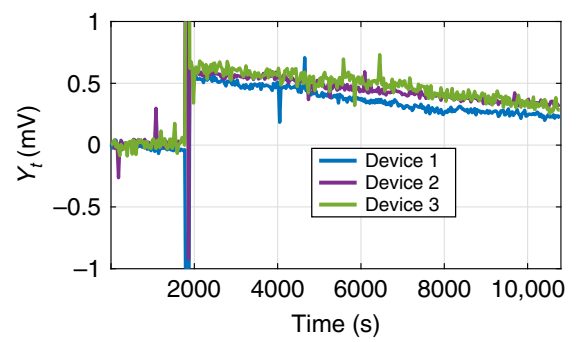

C

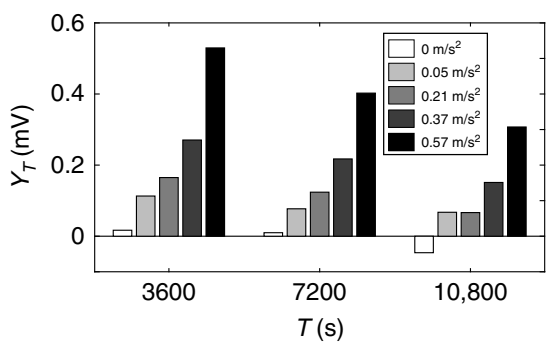

d

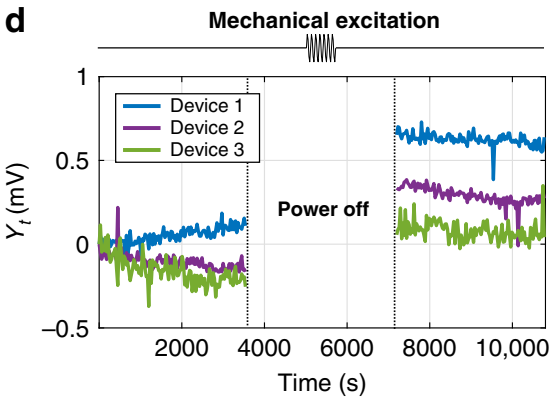

Fig. 5 Self-powered sensing and data logging of mechanical acceleration. a Experimental setup showing a piezoelectric (PVDF) transducer connected to the FN sensor-data-logger chipset. $\mathbf{b}$ Logger response when $58.6 \mathrm{mg}\left(0.57 \mathrm{~m} / \mathrm{s}^{2}\right.$ ) acceleration was applied to the piezo cantilever (gain of $6 \frac{\mathrm{V}}{\mathrm{g}}$ at $75 \mathrm{~Hz}$ resonant frequency) at $72 \mathrm{~Hz}$ for $100 \mathrm{~s}$. c Recorder responses at different readout times for a range of input frequencies. All modulated responses were statistically different from the unmodulated case at all readout times. $\mathbf{d}$ Recorder was powered off in the shaded region. During that time period, vibration table was actuated, which was recorded as evidenced by the recorder value when power supply was turned on.

system depends on the total duration for which the input signal was applied. The initial charge on the floating gate and time to sample are operational parameters as they can be set at run-time, as required by the specific application. Larger time intervals allow the input signal to be integrated over a longer period of time, but it does not change the sensitivity to the signal. For a signal of given action, the measured value decreases as $T$ increases due to resynchronization (Supplementary Fig. 9a). Initializing the device to a higher voltage leads to higher sensitivity but only up to a certain limit (Supplementary Fig. 9b). The reason is that higher sensitivity also leads to faster resynchronization determined by design parameters $k_{1}$ and $k_{2} . k_{1}$ can be tuned by varying the area of tunneling junction and capacitance sizing. We found that increasing the area or lowering the capacitance would increase the sensitivity of the system but only to a certain point (Supplementary Fig. 9c). Beyond this point, the gains are only marginal, at the expense of a larger footprint. Moreover, the capacitance is a function of the tunneling junction area and thus the ratio of area to capacitance is bounded and depends on the permittivity of the insulator. Smaller oxide thickness would decrease $k_{2}$ and sharply increase the sensitivity (Supplementary Fig. 9d). However, at these scales, the effect of other processes like direct tunneling cannot be ignored. Exploring different materials could have significant impact on both $k_{1}$ and $k_{2}$, as they affect the parameters $\alpha$ and $\beta$ (Eqs. (7) and (11)). When the input signal is a single pulse, the time-of-occurrence of the pulse also plays a role in the measured response, as shown in Supplementary Fig. 11. However, this effect is weaker than that of other factors.

The desynchronization-based approach reduces the energy budget required for data logging, we estimate that the proposed device can operate at an energy budget lower than an attojoule while retaining the information for at least $3 \mathrm{~h}$. In standard analog sensor circuits, quiescent current is sourced from a power source for continuous operation. In the proposed device, the quiescent current is the FN quantum tunneling current, which is sourced from the pre-charged capacitor and ambient thermodynamic fluctuations. Hence, no external power source is required for operation. For modulating the sensor, energy is extracted from the signal being sensed. If the energy dissipated at the input signal source (due to finite source impedance) is ignored, the energy budget required to modulate the state of the FN device is $<100 \mathrm{aJ}$. In practice, the energy from the source is spent on charging the capacitor, and for maintaining DC voltage at the source, as described in Supplementary Note 9. For example, when the magnitude of the input signal is $100 \mathrm{mV}, 15 \mathrm{fJ}$ is used for charging a 300-fF input capacitor. The DC input impedance of the proposed device was measured to be $>10^{17} \Omega$; thus, the energy required to maintain a voltage potential of $100 \mathrm{mV}$ for $120 \mathrm{~s}$ is $<100$ aJ. Many signals of interest have power levels greater than this, and can provide sufficient energy for modulating the sensor, provided the system impedance is matched to the source.

However, for time-varying sensor signals, a more efficient power transfer or sensing is achieved as some energy stored in reactive elements like capacitors can be recovered. In Supplementary Fig. 10, an equivalent circuit model corresponding to the FN tunneling device is shown along with a simplified sensortransducer equivalent model. We note that the input impedance of the recorder is predominantly capacitive and the only dissipative factor arising during sensing/data logging is due to the FN tunneling current. The equivalent circuit model allows estimation of the power dissipated by a device that is excited by an arbitrary sensor signal. In Supplementary Note 9, we show that a broadband AC input signal with upper cutoff frequency of $1 \mathrm{kHz}$ and amplitude of $100 \mathrm{mV}$ has an estimated energy dissipation by the system of $5 \mathrm{aJ}$ for an event lasting $100 \mathrm{~s}$.

Using FN quantum tunneling to implement the dynamical system has some key advantages. Its stability allowed us to create a pair of synchronized devices which is compensated for environmental variations. Its predictability was used for modeling, and we were able to derive a recorder response model that matched experimental data with $98.8 \%$ accuracy. Its dynamics follow a $1 / \log (t)$ characteristic, which yields a long operational life. The non-linear response leads to rectification of input signals and offers an opportunity for time stamping and reconstruction. A more rigorous and theoretic investigation into the use of dynamical systems for information reconstruction will be the topic of future research. 
At its core, the proposed device consists of four capacitors and two transistors $(4 \mathrm{C}-2 \mathrm{~T})$, and can be implemented on any standard CMOS process. The current design is a proof-of-concept and is not optimized for sensitivity or form factor. Modeling analysis in Supplementary Fig. 9 shows that both of these parameters can be improved by minimizing the capacitance, while maintaining the capacitance ratio $\left(C_{\mathrm{R}}\right)$. To achieve this, an optimum balance between the input capacitor, decoupling capacitor and parasitic capacitance at the poly-substrate tunneling junction needs to be obtained. Better matching of the sensor and reference nodes (tunneling junctions, capacitors and readout circuits) using advanced analog layout techniques should be able to reduce the operational noise in the recorder and thereby increase the data retention capacity. Readout and common-mode noise can be further reduced by implementing a low-noise on-chip instrumentation amplifier. Multiple units of independent recorders could be used to increase the SNR of the recordings.

Any passive sensor that is capable of transducing a physical signal into an electrical signal (voltage or current) can be interfaced with our system. These include piezoelectric transducers, photodiodes, radio-frequency antennas, thermocouples, triboelectric generators, etc. Passive sensors like strain gauges, that do not directly produce electrical output but instead effect a change in resistance, are not compatible with our system. Similarly, many chemical transducers like dopamine sensors that require an activation voltage (external biasing or power) are also not applicable for self-powered data logging. However, chemical sensors like amperometric glucose sensor that have the ability to generate electrical charge during the process of sensing should work with our system. In addition, there can be practical issues in measuring certain types of signals. For example, the limited action generated by signals like neural action-potentials may not be measurable due to resynchronization and system-noise. Finally, the proposed recorder could be directly integrated with FET (field-effect transistor)-based sensors ${ }^{22-24}$, which have been developed for a wide range of applications. As there are no extrinsic powering requirements, there is the potential of integrating these devices on "smart dust" platforms as well"25,26.

In conclusion, we have described a self-powered sensor-datalogger device that records a cumulative measure of the sensor signal intensity over its entire duration. To achieve this, we designed a pair of synchronized dynamical systems whose trajectories are modulated by an external signal. The modulation leaves its trace by desynchronizing one of the synchronized pairs. The total cumulative measure or action is stored as a dynamical state which is then measured at a later instant of time. The selfpowered dynamical system was designed by exploiting the physics of FN quantum tunneling in floating-gate transistors. We modeled the response of our system to an arbitrary signal and verified the model experimentally. We also demonstrated the self-powered sensing capabilities of our device by logging mechanical vibration signals produced by a small piezoelectric transducer, while being disconnected from any external power source.

\section{Methods}

One-time programming. For each node of each recorder, the readout voltage was programmed to around $3 \mathrm{~V}$ while the tunneling node was operating in the tunneling regime. This was achieved through a combination of tunneling and injection. Specifically, chip power supply (VDD) was set to $7 \mathrm{~V}$, input pins to $5 \mathrm{~V}$ and the program tunneling pin (Vprog) was gradually increased to $23 \mathrm{~V}$. Around $12-13 \mathrm{~V}$, the tunneling node's potential would start increasing. The coupled readout node's potential would also increase. When the readout potential went over $4.5 \mathrm{~V}$, electrons would start injecting into the readout floating gate, thus ensuring its potential was clamped below $5 \mathrm{~V}$. After this initial programming, VDD was set to $5 \mathrm{~V}$ for the rest of the experiments.
Calibration. After one-time programming, input was set to $0 \mathrm{~V}$, Vprog to $21.5 \mathrm{~V}$ for $1 \mathrm{~min}$ and then the floating gate was allowed to discharge naturally. Readout voltages for the sensor and reference nodes were measured every $30 \mathrm{~s}$, for $3 \mathrm{~h}$. The rate of discharge for each node was calculated; and a state where the tunneling rates would be equal was chosen as the initial synchronization point for the remainder of the experiments.

Initialization. Before the start of each experiment, floating gates were initialized to the initial synchronization point, estimated in the previous section. This was done by either setting the input to stable DC point through a digital to analog converter (DAC) or if the DAC value needed was beyond its output limit, then the potential would be increased by setting Vprog pin to $21 \mathrm{~V}$.

Model derivation. FN tunneling current density $J_{\mathrm{FN}}$ across a triangular barrier can be expressed as a function of the electric field $E$ across the barrier ${ }^{19}$ :

$$
J_{\mathrm{FN}}(E)=\alpha E^{2} \exp (-\beta / E)
$$

where $\alpha$ and $\beta$ are process and device specific parameters ${ }^{19}$.

Thus, for a tunneling junction with cross-sectional area $A$ and thickness $t_{\mathrm{ox}}$, the tunneling current $I_{\mathrm{FN}}$ for a time-varying voltage $V_{t}$ is given by

$$
I_{\mathrm{FN}}\left(V_{t}\right)=A \alpha\left(V_{t} / t_{\mathrm{ox}}\right)^{2} \exp \left(-\beta t_{\mathrm{ox}} / V_{t}\right) .
$$

Referring to the equivalent circuit in Fig. $2 \mathrm{a}$, the dynamical system model when the sensing signal $x_{t}$ is absent is given by

$$
I_{\mathrm{FN}}\left(V_{t}\right)=-C_{\text {total }} \frac{d V_{t}}{d t},
$$

where $C_{\text {total }}=C+C_{\text {in }}$ is the total capacitance at the floating-gate node. The solution of the equation can be expressed as :

$$
V_{t}=\frac{k_{2}}{\log \left(k_{1} t+k_{0}\right)},
$$

where

$$
k_{1}=\frac{A \alpha \beta}{C t_{\mathrm{ox}}} \quad, k_{2}=\beta t_{\mathrm{ox}}
$$

depend on material properties and device structure, while

$$
k_{0}=\exp \left(\frac{k_{2}}{V_{0}}\right)
$$

depends on the initial conditions.

Now, let

$$
\mathrm{f}\left(V_{t}\right)=-\frac{I\left(V_{t}\right)}{C_{\text {total }}}=-\frac{k_{1}}{k_{2}} V_{t}^{2} \exp \left(\frac{-k_{2}}{V_{t}}\right) .
$$

Desynchronization between the sensor and reference nodes shown in Fig. 2a occurs because of differences in rates of tunneling, which are caused by differences in electric potentials across the respective floating gates

$$
\begin{aligned}
\frac{d Y_{t}}{d t} & =\frac{I_{\mathrm{FN}}\left(V_{t}^{S}\right)}{C_{\text {total }}}-\frac{I_{\mathrm{FN}}\left(V_{t}^{R}\right)}{C_{\text {total }}} \\
& =\mathrm{f}\left(V_{t}^{R}\right)-\mathrm{f}\left(V_{t}^{S}\right) .
\end{aligned}
$$

The reference node $V_{t}^{R}$ follows the dynamics of Eq. (10) as it is not under the action of an external field. Thus, $V_{t}^{R}=V_{t}$. The potential across the sensing node is given by how much it has desynchronized from the reference node $\left(V_{t}^{R}-Y_{t}\right)$ and the effect of the external field, $x_{t}$, through the input capacitor $C_{\text {in }}$.

$$
V_{t}^{S}=V_{t}+C_{\mathrm{R}} x_{t}-Y_{t}
$$

where $C_{\mathrm{R}}$ is the coupling ratio due to capacitive divider formed by $C_{\mathrm{in}}$ and $C_{\mathrm{fg}}$.

$$
C_{\mathrm{R}}=\frac{C_{\text {in }}}{C_{\text {total }}} ; C_{\text {total }}=C_{\text {in }}+C_{\mathrm{FG} 1}+C_{\mathrm{C}} \| C_{\mathrm{FG} 2} .
$$

Substituting $V_{t}^{R}$ and $V_{t}^{R}$ in Eq. (13),

$$
\frac{d Y_{t}}{d t}=\mathrm{f}\left(V_{t}\right)-\mathrm{f}\left(V_{t}+C_{\mathrm{R}} x_{t}-Y_{t}\right)
$$

The above equation is the constitutive differential equation and can be solved using numerical methods for any input signal. To obtain an explicit expression for estimating the response $Y_{t}$, we assume that $Y_{t} \ll V_{t}$ and $\mathrm{E}\left(x_{t}\right)=0$ for all $t$, and use Taylor series expansion with first-order approximation.

$$
\begin{aligned}
& \frac{d Y_{t}}{d t}=\mathrm{f}\left(V_{t}\right)-\mathrm{f}\left(V_{t}+C_{\mathrm{R}} x_{t}\right)+\frac{d\left(\mathrm{f}\left(V_{t}\right)\right)}{\mathrm{d} V_{t}} Y_{t} \\
& \frac{d Y_{t}}{d t}-\frac{d\left(\mathrm{f}\left(V_{t}\right)\right)}{d V_{t}} Y_{t}=\mathrm{f}\left(V_{t}\right)-\mathrm{f}\left(V_{t}+C_{\mathrm{R}} x_{t}\right) .
\end{aligned}
$$


Multiplying both sides of Eq. (17) by $1 / \mathrm{f}\left(V_{t}\right)$, substituting $\mathrm{d} V_{t}=\mathrm{f}\left(V_{t}\right) \mathrm{d} t$ (from Eqs. (9) and (12)) and simplifying:

$$
\begin{aligned}
\frac{d Y_{t}}{\mathrm{f}\left(V_{t}\right) d t}-\frac{d\left(\mathrm{f}\left(V_{t}\right)\right)}{\mathrm{f}\left(V_{t}\right)^{2} \mathrm{~d} t} Y_{t} & =\frac{1}{\mathrm{f}\left(V_{t}\right)}\left(\mathrm{f}\left(V_{t}\right)-\mathrm{f}\left(V_{t}+C_{\mathrm{R}} x_{t}\right)\right) \\
\frac{d}{d t}\left(\frac{Y_{t}}{\mathrm{f}\left(V_{t}\right)}\right) & =1-\frac{\mathrm{f}\left(V_{t}+C_{\mathrm{R}} x_{t}\right)}{\mathrm{f}\left(V_{t}\right)} .
\end{aligned}
$$

Integrating both sides with respect to $\mathrm{d} t$ between the limits 0 and $T$ :

$$
\begin{aligned}
\frac{Y_{T}}{\mathrm{f}\left(V_{T}\right)}-\frac{Y_{0}}{\mathrm{f}\left(V_{0}\right)} & =\int_{0}^{T}\left(1-\frac{\mathrm{f}\left(V_{t}+C_{\mathrm{R}} x_{t}\right)}{\mathrm{f}\left(V_{t}\right)}\right) d t \\
\frac{Y_{T}}{\mathrm{f}\left(V_{T}\right)} & =\int_{0}^{T}\left(1-\frac{\mathrm{f}\left(V_{t}+C_{\mathrm{R}} x_{t}\right)}{\mathrm{f}\left(V_{t}\right)}\right) d t \\
Y_{T} & =\mathrm{f}\left(V_{T}\right) \int_{0}^{T}\left(1-\frac{\mathrm{f}\left(V_{t}+C_{\mathrm{R}} x_{t}\right)}{\mathrm{f}\left(V_{t}\right)}\right) d t .
\end{aligned}
$$

Substituting $\mathrm{f}\left(V_{t}\right)$ from Eq. (12) into Eq. (19),

$$
Y_{T}=\frac{k_{1}}{k_{2}} V_{T}^{2} \exp \left(\frac{-k_{2}}{V_{T}}\right) \int_{0}^{T}\left[\left(1+\frac{C_{\mathrm{R}} x_{t}}{V_{t}}\right)^{2} \exp \left(\frac{k_{2} C_{\mathrm{R}} x_{t}}{V_{t}\left(V_{t}+C_{\mathrm{R}} x_{t}\right)}\right)-1\right] \mathrm{d} t .
$$

Reporting summary. Further information on research design is available in the Nature Research Reporting Summary linked to this article.

\section{Data availability}

All the software and experimental data used for generating the figures have been deposited in a public repository (https://doi.org/10.6084/m9.figshare.12814592.v1) ${ }^{27}$

Received: 21 April 2020; Accepted: 1 October 2020;

Published online: 28 October 2020

\section{References}

1. Mathews, I., Kelly, G., King, P. J. \& Frizzell, R. Gaas solar cells for indoor light harvesting. In 2014 IEEE 40th Photovoltaic Specialist Conference (PVSC), 0510-0513 (IEEE, Denver, CO, USA, 2014).

2. Wang, Z. L. \& Song, J. Piezoelectric nanogenerators based on zinc oxide nanowire arrays. Science 312, 242-246 (2006).

3. Torah, R. et al. Self-powered autonomous wireless sensor node using vibration energy harvesting. Meas. Sci. Technol. 19, 125202 (2008).

4. McEvoy, M. A. \& Correll, N. Materials that couple sensing, actuation, computation, and communication. Science 347, 1261689 (2015).

5. Huang, C. \& Chakrabartty, S. An asynchronous analog self-powered cmos sensor-data-logger with a $13.56 \mathrm{mhz}$ rf programming interface. IEEE J. SolidState Circuits 47, 476-489 (2011).

6. Chakrabartty, S. Self-powered sensor. US patent 7,757,565 (2010)

7. Zhou, L., Abraham, A. C., Tang, S. Y. \& Chakrabartty, S. A 5 nw quasi-linear cmos hot-electron injector for self-powered monitoring of biomechanical strain variations. IEEE Trans. Biomed. Circuits Syst. 10, 1143-1151 (2016).

8. Åkerman, J. Toward a universal memory. Science 308, 508-510 (2005).

9. Auciello, O., Scott, J. F. \& Ramesh, R. et al. The physics of ferroelectric memories. Phys. Today 51, 22-27 (1998).

10. Chua, L. Memristor-the missing circuit element. IEEE Trans. Circuit Theory 18, 507-519 (1971)

11. Ramadass, Y. K. \& Chandrakasan, A. P. A battery-less thermoelectric energy harvesting interface circuit with $35 \mathrm{mv}$ startup voltage. IEEE J. Solid-State Circuits 46, 333-341 (2011).

12. Mercier, P. P., Lysaght, A. C., Bandyopadhyay, S., Chandrakasan, A. P. \& Stankovic, K. M. Energy extraction from the biologic battery in the inner ear. Nat. Biotechnol. 30, 1240 (2012).

13. Texas Instruments. bq25505 - ultra low-power boost charger with battery management and autonomous power multiplexer for primary battery in energy harvester applications. SLUSBJ3F. http://www.ti.com/lit/ds/symlink/ bq25505.pdf. (2019).

14. Appeltant, L. et al. Information processing using a single dynamical node as complex system. Nat. Commun. 2, 1-6 (2011).

15. Dambre, J., Verstraeten, D., Schrauwen, B. \& Massar, S. Information processing capacity of dynamical systems. Sci. Rep. 2, 1-7 (2012)

16. Ganguli, S., Huh, D. \& Sompolinsky, H. Memory traces in dynamical systems. Proc. Natl Acad. Sci. USA 105, 18970-18975 (2008).

17. Mehta, D., Raman, B. \& Chakrabartty, S. Differential Fowler-Nordheim tunneling dynamical system for attojoule sensing and recording. In 2019 IEEE
International Symposium on Circuits and Systems (ISCAS) 1-5 https://doi.org/ 10.1109/ISCAS.2019.8702685 (IEEE, Sapporo, Japan, 2019).

18. Zhou, L. \& Chakrabartty, S. Self-powered timekeeping and synchronization using Fowler-Nordheim tunneling-based floating-gate integrators. IEEE Trans. Electron Devices 64, 1254-1260 (2017).

19. Lenzlinger, M. \& Snow, E. Fowler-Nordheim tunneling into thermally grown sio2. J. Appl. Phys. 40, 278-283 (1969).

20. Harrison, R. R., Bragg, J. A., Hasler, P., Minch, B. A. \& Deweerth, S. P. A cmos programmable analog memory-cell array using floating-gate circuits. IEEE Trans. Circuits Syst. II: Analog Digit. Signal Process. 48, 4-11 (2001).

21. Roundy, S. On the effectiveness of vibration-based energy harvesting. J. Intell. Mater. Syst. Struct. 16, 809-823 (2005).

22. Middelhoek, S. Celebration of the tenth transducers conference: the past, present and future of transducer research and development. Sens. Actuators A Phys. 82, 2-23 (2000)

23. Bergveld, $P$. Thirty years of isfetology: what happened in the past 30 years and what may happen in the next 30 years. Sens. Actuators B Chem. 88, 1-20 (2003).

24. Torsi, L., Magliulo, M., Manoli, K. \& Palazzo, G. Organic field-effect transistor sensors: a tutorial review. Chem. Soc. Rev. 42, 8612-8628 (2013).

25. Warneke, B., Last, M., Liebowitz, B. \& Pister, K. S. Smart dust: communicating with a cubic-millimeter computer. Computer 34, 44-51 (2001).

26. Seo, D. et al. Wireless recording in the peripheral nervous system with ultrasonic neural dust. Neuron 91, 529-539 (2016).

27. Mehta, D. \& Chakrabartty, S. Self-powered analog sensor-data-logger experimental data. https://doi.org/10.6084/m9.figshare.12814592.v1 (2020).

\section{Acknowledgements}

This work was supported in part by NIH research grants 1R21EY028362-01 and R21AR075242-01. The authors acknowledge the help and resources provided by Prof. Srikanth Singamaneni and Prashant Gupta in acquiring micrographs of the fabricated chips. We thank Dr. Liang Zhou for useful discussions regarding quantum tunneling dynamics and circuit design. Owen Pochettino is acknowledged for helping build a chip testing station.

\section{Author contributions}

D.M., K.A. and S.C. conceived the project. D.M. and S.C. designed the experiments. D.M. and K.A. developed the instrumentation for data collection. D.M. performed the experiments and collected the data. D.M. and K.A. analyzed the data and generated the figures. S.C. supervised all aspects of the work. All authors contributed to the writing of the manuscript

\section{Competing Interests}

The authors declare no competing interests.

\section{Additional information}

Supplementary information is available for this paper at https://doi.org/10.1038/s41467 020-19292-w.

Correspondence and requests for materials should be addressed to S.C.

Peer review information Nature Communications thanks Michel Maharbiz and the other, anonymous reviewer(s) for their contribution to the peer review of this work. Peer reviewer reports are available.

Reprints and permission information is available at http://www.nature.com/reprint

Publisher's note Springer Nature remains neutral with regard to jurisdictional claims in published maps and institutional affiliations.

Open Access This article is licensed under a Creative Commons Attribution 4.0 International License, which permits use, sharing, adaptation, distribution and reproduction in any medium or format, as long as you give appropriate credit to the original author(s) and the source, provide a link to the Creative Commons license, and indicate if changes were made. The images or other third party material in this article are included in the article's Creative Commons license, unless indicated otherwise in a credit line to the material. If material is not included in the article's Creative Commons license and your intended use is not permitted by statutory regulation or exceeds the permitted use, you will need to obtain permission directly from the copyright holder. To view a copy of this license, visit http://creativecommons.org/ licenses/by/4.0/

(c) The Author(s) 2020 